\title{
Transcriptomic epidemiology of smoking: the effect of smoking on gene expression in lymphocytes
}

\author{
Jac C Charlesworth *1,2, Joanne E Curran', Matthew P Johnson'1, Harald HH Göring1', Thomas D Dyer1, \\ Vincent P Diego ${ }^{1}$, Jack W Kent Jr' , Michael C Mahaney ${ }^{1}$, Laura Almasy' ${ }^{1}$ Jean W MacCluer ${ }^{1}$, Eric K Moses ${ }^{1}$ and \\ John Blangero'
}

\begin{abstract}
Background: This investigation offers insights into system-wide pathological processes induced in response to cigarette smoke exposure by determining its influences at the gene expression level.

Methods: We obtained genome-wide quantitative transcriptional profiles from 1,240 individuals from the San Antonio Family Heart Study, including 297 current smokers. Using lymphocyte samples, we identified 20,413 transcripts with significantly detectable expression levels, including both known and predicted genes. Correlation between smoking and gene expression levels was determined using a regression model that allows for residual genetic effects.

Results: With a conservative false-discovery rate of $5 \%$ we identified 323 unique genes (342 transcripts) whose expression levels were significantly correlated with smoking behavior. These genes showed significant overrepresentation within a range of functional categories that correspond well with known smoking-related pathologies, including immune response, cell death, cancer, natural killer cell signaling and xenobiotic metabolism.

Conclusions: Our results indicate that not only individual genes but entire networks of gene interaction are influenced by cigarette smoking. This is the largest in vivo transcriptomic epidemiological study of smoking to date and reveals the significant and comprehensive influence of cigarette smoke, as an environmental variable, on the expression of genes. The central importance of this manuscript is to provide a summary of the relationships between gene expression and smoking in this exceptionally large cross-sectional data set.
\end{abstract}

\section{Background}

Tobacco use is responsible for more than 5 million deaths per year [1] and is the leading preventable cause of premature death worldwide. Smoking is known to have a major impact on human health, adversely affecting almost every organ. Exposure to cigarette smoke increases the risk of many diseases, including a wide range of cancers (from lung to pancreatic cancer), cardiovascular diseases (including atherosclerosis and coronary heart disease), a range of respiratory diseases (including chronic obstructive pulmonary disease and pneumonia), as well as various other adverse health effects such as

* Correspondence: jac.charlesworth@utas.edu.au

1 Department of Genetics, Southwest Foundation for Biomedical Research, P.O. Box 760549, San Antonio, TX, USA

Full list of author information is available at the end of the article increased risk of cataracts, infection and poor wound healing, and is generally detrimental to the overall health of individuals who smoke [2-8].

Investigating the influence of cigarette smoke exposure on health is a highly complex problem. The particulate and vapor phase of cigarette smoke contains in excess of 4,000 compounds, including five known human carcinogens and many toxic agents $[9,10]$. These toxins enter the bloodstream, via the pulmonary alveoli, and are distributed throughout the body. The widespread organ damage in active smokers reflects the systemic distribution of these compounds and the variety of cell types that are exposed. Studies of the effects of cigarette smoking have employed a variety of approaches to reduce the complexity of the problem, such as studying animal models or individual cell types in vitro that are exposed to 'standard- 
ized' measures of cigarette smoke, or to individual components of the particulate or vapor phases. However, no one model is able to capture the biological heterogeneity of the effects.

This study utilized large-scale genome-wide expression profiling as an alternative approach to determine the systemic influence of cigarette smoke, as an environmental exposure, on human physiology and health. Previous studies of gene expression as influenced by smoking have been seriously limited in size [11-21] with the largest of the in vivo studies including only 42 smokers and 43 nonsmokers [15]. The small sample sizes and general lack of power have resulted in little concordance between these studies. Our hypothesis was that, given a sufficiently large set of related individuals, a stable and interpretable pattern of gene expression alterations attributable to cigarette smoke exposure may be obtained. In addition, a large and complex dataset allows for both the investigation of significant results at the individual gene level and provides the ability to determine elaborate networks of alteration. Studying these patterns of expression alteration in response to cigarette smoke exposure may provide the key to understanding the pathogenesis of many of the adverse health effects attributable to smoking and the interactions between them.

\section{Methods}

\section{Ethics statement}

All protocols were approved by the Institutional Review Board of the University of Texas Health Science Center at San Antonio. Participants gave informed consent and all investigation were conducted according to the principles expressed in the Declaration of Helsinki

\section{Study population}

This investigation was conducted as part of the SAFHS, initiated in 1992 to investigate the genetics of cardiovascular disease and its risk factors in Mexican Americans [22]. Ascertainment occurred by way of adult probands selected at random, without regard to presence or absence of disease, from the Mexican American community in San Antonio, Texas. To ensure large, multigenerational pedigrees, probands had to have at least 6 ageeligible offspring and/or siblings living in San Antonio. All first, second, and third degree relatives of the proband and of the proband's spouse, aged 16 years or above, were eligible to participate in the study. More than 1,400 individuals from 42 extended families were recruited [22]. Reported family relationships were verified using the computer program PREST [23], based on autosomal genotype data, and corrections to the familial relationships were applied where required. Existing blood samples and phenotype data from the SAFHS were utilized in this investigation.

\section{Assessment of smoking status}

Smoking status was assessed by structured interview conducted during the first clinic visit between 1991 and 1995, the same time point as the lymphocyte collection for the expression profiling. Data collected included current smoking status (smoker or non-smoker) as well as an estimate of cigarettes smoked per day, all by self report. No data were available on duration or former smoking status. In addition, serum cotinine levels were measured using a commercially available ELISA assay (BioQuant, San Diego, California). Serum for the cotinine assay was obtained during the first clinic visit but was only available for 981 of the 1,240 individuals with expression profiles.

\section{Expression profiling}

The expression profiling methodology is described, in detail, in Göring et al. (2007) [24]. In brief, frozen lymphocyte samples were available from 1,240 individuals, collected during their first clinic visit between 1991 and 1995, after an overnight fast, in EDTA tubes. Lymphocytes were isolated from a $10 \mathrm{ml}$ sample using Histopaque (Sigma Chemical Co., St. Louis, MO), following the suggested protocol of the manufacturer, washed, and stored in a freeze media in liquid nitrogen.

Total RNA was isolated using a modified procedure of the QIAGEN RNeasy' 96 protocol for isolation of total RNA from animal cells using spin technology (QIAGEN Inc., Valencia, CA), and a total of $500 \mathrm{ng}$ total RNA dried down and stored at $-20^{\circ} \mathrm{C}$. Anti-sense RNA (aRNA) was synthesized, amplified and purified using the Ambion MessageAmp II Amplification Kit (Ambion, Austin, TX) following the Illumina Sentrix Array Matrix 96-well expression protocol (Illumina Inc., San Diego, CA). Synthesized cDNA samples were purified using QIAGEN's QIAquick 96 PCR purification supplementary protocol for spin technology (QIAGEN document QQ01.doc, October 2001). Biotin-16-UTP (Roche, Germany) labeled aRNA was synthesized using Ambion's proprietary MEGAscript in vitro transcription (IVT) technology and T7 RNA Polymerase. Purification of aRNA samples was performed using QIAGEN's RNeasy 96 protocol for RNA cleanup using spin technology, and a total of $1.5 \mu \mathrm{g}$ aRNA was dried and stored at $-20^{\circ} \mathrm{C}$ prior to sample hybridization.

Hybridization of aRNA to Illumina Sentrix Human Whole Genome (WG-6) Series I BeadChips and subsequent washing, blocking and detecting were performed using Illumina's BeadChip $6 \times 2$ protocol, as described in Göring et al. [24]. Samples were scanned on the Illumina BeadArray $^{\text {ma }}$ 500GX Reader using Illumina BeadScan image data acquisition software (version 2.3.0.13). Illumina BeadStudio software (version 1.5.0.34) was used for preliminary data analysis, with a standard background normalization, to generate an output file for statistical 
analysis. In total we interrogated 47,289 unique transcripts: 22,151 probes (47\%) are targeted at Reference Sequence (RefSeq) transcripts, and the remaining 25,128 probes $(53 \%)$ correspond to other, generally less well characterized transcripts (including predicted genes) [24].

\section{Identification of expressed transcripts}

In order to identify transcripts that exhibited sufficient quantitative expression in lymphocytes, the distribution of expression values for a given transcript was compared to the distribution of the expression values of the controls that are imbedded in each chip. For each transcript, we performed a $x^{2}$ "tail" test of whether there was a significant excess of samples with values above the 95th percentile of the control null distribution. This test was used because it allows detection of even those transcripts that are clearly present above baseline levels in only a subset of individuals, while not being detectable above baseline levels in most individuals. Using a false discovery rate of 0.05 , we identified 20,413 transcripts that exhibited significant expression by this criterion.

\section{Standardization of expression values}

To minimize the influence of overall signal levels, which may reflect RNA quantity and quality rather than a true biological difference between individuals, abundance values of all 20,413 retained transcripts were first standardized by $\mathrm{z}$-scoring within individuals (using decile percentage bins of transcripts, grouped by average logtransformed raw signals across individuals), followed by linear regression against individual-specific average logtransformed raw signal and its squared value. Lastly, for each transcript, we directly normalized these residual expression scores by employing an inverse Gaussian transformation across individuals, to ensure that the assumptions underlying variance components-based analyses were not violated. This conservative procedure results in normalized expression phenotypes that are comparable between individuals and across transcripts.

\section{Statistical Analyses}

All statistical analyses on related individuals were performed using variance components-based methodology and software package SOLAR 4.1 [25]. To ensure that the assumption of a multivariate normal phenotypic distribution was not violated, we subjected all phenotypes to an exact inverse normalization procedure prior to analysis. We tested for association between smoking and gene expression levels using a regression model that allows for residual genetic effects, as implemented in SOLAR. In this approach, smoking was treated as a covariate for a given transcript's expression level. A likelihood ratio statistic was used to formally test the hypothesis that smok- ing was correlated with gene expression levels. This test was performed conditionally upon other covariate effects including those of sex, age, and their interactions. A false discovery rate approach [26] was utilized to deal with the major issue of multiple testing. We employed a rigorous FDR of 0.05 for all analyses.

We used combined discrete-continuous bivariate modeling analysis [27] to determine the environmental and genetic correlations between smoking (as a discrete trait) and expression of any given transcript (as a quantitative trait). Formal likelihood-based tests were used to test the difference of the genetic $\left(\rho_{\mathrm{g}}\right)$ and environmental correlation $\left(\rho_{\mathrm{e}}\right)$ from zero.

\section{Pathway and Networking Analysis}

The 342 transcripts meeting the FDR of 0.05 criteria were analyzed using Ingenuity Pathways Analysis version 6.3 (Ingenuity ${ }^{\circ}$ Systems, http://www.ingenuity.com). There were 322 transcripts that mapped to known genes, including two cases where three significant transcripts corresponded to one gene (GNLY and PID1) and fifteen cases where there were two significant transcripts within a gene (BCN2, C9ORF142, CDH23, CLEC10A, FLJ16686, LGR6, LMNA, LYPD2, MMP25, NCF4, SH2D3C, SNTB2, SSBP3, TCF7L2, TRA@ and ZAK). In total there were 303 unique smoking correlated genes identified by IPA from the list of 342 significant transcripts. There were 20 transcripts that were unidentified and not included in the analyses, predominantly because their identifier had been retired or corresponded to a pseudogene (entrez gene IDs $28804,80022,255519$ ) or hypothetical protein not yet described in the literature. All 323 unique identifiers (303 known and 20 unknown) are shown in Additional File 1.

The right-tailed Fisher's exact test was used to calculate a p-value determining the probability that each biological function and/or disease assigned to that dataset was due to chance alone. This p-value is calculated by comparing the number of user-imported genes in a given function or pathway relative to the total number of occurrences of these genes in all functional/pathway annotations stored in the knowledge base for the reference set. We used the entire set of 20,413 transcripts that were significantly detected in lymphocytes in our study [24] as the reference set for this investigation.

Our genes of interest were overlaid onto a global molecular network developed from literature reported connectivity recorded in the Ingenuity Pathways Knowledge Base, allowing the generation of gene networks; graphical representation of the molecular relationships between genes/gene products. All interactions between genes and other molecules in these networks are supported by peer reviewed publication. 


\section{Accession Number}

Raw expression values (of all transcripts on the microarray) and normalized expression values (of all 19,648 analyzed autosomal transcripts), along with information on sex and age are available under accession number ETABM-305 on the ArrayExpress website http:// www.ebi.ac.uk/arrayexpress/.

\section{Results}

\section{Study Summary}

For this study, transcriptional profiles were obtained from 1,240 Mexican American individuals from the San Antonio Family Heart Study (SAFHS). This dataset contained 1,154 individuals from 46 pedigrees and an additional 86 singletons. There were 734 females and 506 males in the sample, with a mean age of 39.3 years $(\mathrm{SD}=$ 16.7 years). Ages ranged between 16 and 94 years. For each sample, 47,289 transcripts were interrogated using the Sentrix Human- 6 expression BeadChip supplied by Illumina (San Diego, CA). We were able to significantly detect 20,413 expressed transcripts in lymphocytes, $62.5 \%$ of these corresponding to known genes [24].

The prevalence of smoking in the dataset was $24 \%$, with 297 current smokers. Using the genome-wide transcriptional profile dataset, we tested for correlations of gene expression in lymphocytes with a discrete measure of current smoking behavior, assessed by questionnaire. With a conservative false-discovery rate of $5 \%$, corresponding to an observed nominal p-value of $<0.001$, we identified 342 transcripts whose expression levels were significantly correlated with smoking, 110 (32.2\%) with positive correlations and $232(67.8 \%)$ negatively correlated. These 342 transcripts correspond to 323 unique genes. Details of this set of genes and the correlation of expression with smoking are provided in Additional File 1. Increasing the FDR to $10 \%$ increased the number of significant transcripts to 474 , corresponding to an observed nominal p-value of $<0.0028$.

\section{Validation of the phenotype}

A quantitative measure of average cigarettes per day was available in this study, but was deemed less-reliable than the discrete trait, owing to bias introduced by self-report [28]. However, analyses using the quantitative measure did validate the set of smoking-correlated transcripts identified using the more conservative discrete trait, with a tetrachoric correlation of $0.905 \pm 0.012$ between transcripts significantly correlated with each measure at a $5 \%$ FDR.

In addition, plasma cotinine levels were available for a subset $(79.1 \%)$ of the 1,240 studied individuals. Cotinine is a nicotine metabolite that is often used as a quantitative measure of cigarette smoking; however cotinine levels are subject to both genetic and environmental variation [29].
Using a plasma cotinine level of $\geq 20$ for smoking and $\geq 300$ for heavy smoking we only identified 17 individuals whose self-reported smoking status was clearly misclassified. The tetrachoric correlation between plasma cotinine levels and self-reported smoking status was extremely high $(0.979 \pm 0.007)$.

\section{Functional Annotation Analysis}

In order to identify specific pathways and functional assignments involved in the response to smoking, we performed a series of formal pathway analyses. The 342 transcripts meeting the FDR of 0.05 criteria were analyzed using Ingenuity Pathways Analysis (IPA) version 6.3 (Ingenuity Systems, http://www.ingenuity.com). A total of 214 of the smoking-correlated genes included information on functions and/or canonical pathways from the published literature, which was used to identify overrepresentation of smoking correlated genes within known categories of functional assignments (such as immune response), and to develop hypotheses of gene action in the context of wider biological relationships. The most significant functional assignments included cell cytotoxicity $\left(\mathrm{p}=3.7 \times 10^{-10}\right)$, immune response $\left(\mathrm{p}=2.2 \times 10^{-7}\right)$, and tumorigenesis $\left(\mathrm{p}=1.2 \times 10^{-6}\right)$. The most significant functional assignments are shown in Table 1 , along with the $\mathrm{p}$-value for the significance of each assignment and the total number of smoking correlated genes within each category. These are documented in more detail in Additional File 1, which includes the functional and canonical pathway assignments for each individual transcript within the dataset.

There were several highly significant functional categories involved in various aspects of cell death, including 17 genes involved in cell cytotoxicity (CD38, CD300A, FASLG, FCGR3A, FCGR3B, GZMA, GZMB, KLRB1, KLRD1, KLRF1, KLRK1, PRF1, PTGDR, PTPN6, SLAMF7, SPN, TNFRSF8; $\left.\mathrm{p}=3.7 \times 10^{-10}\right)$ and 11 in cell lysis (ABCB1, CX3CR1, FASLG, FCGR3A, GNLY, GZMA, GZMB, KLRB1, KLRD1, KLRK1, PRF1; p = $1.2 \times 10^{-7}$ ) that were all significantly negatively correlated with smoking. In total there were 66 significant transcripts for genes involved in various aspects of cell death $(\mathrm{p}=1.1 \times$ $\left.10^{-6}\right), 47$ negatively correlated and 19 positively correlated with smoking.

There were 38 smoking-correlated genes involved in immune response $\left(\mathrm{p}=2.2 \times 10^{-7}\right)$, including 30 negatively correlated with smoking $(A D A, C 3, C D 38, C D 247$, CD300A, CST7, CTSC, CTSL1, CTSW, CX3CR1, FASLG, FCGR3A, FCGR3B, GFI1, GNLY, GZMA, GZMB, IL18RAP, IL2RB, ITGAX, KLRD1, KLRF1, KLRK1, PIK3CG, PRF1, SPN, SPON2, TGFBR3, TNFRSF14 and TRG@) and eight positively correlated genes (CLEC5A, EBI2, IGHE, MGST1, NCF4, RNASE2, SLAMF1 and TPSAB1). 
Table 1: The most highly significant functional assignments for the set of smoking correlated genes

\begin{tabular}{|c|c|c|}
\hline Function Annotation & P-value & Number of genes \\
\hline Cell cytotoxicity & $3.7 \times 10^{-10}$ & 17 \\
\hline Proliferation of cells & $2.1 \times 10^{-9}$ & 65 \\
\hline Activation of cells & $1.7 \times 10^{-8}$ & 31 \\
\hline Cell movement & $2.9 \times 10^{-8}$ & 35 \\
\hline Lysis of cells & $1.2 \times 10^{-7}$ & 11 \\
\hline Immune response & $2.2 \times 10^{-7}$ & 38 \\
\hline Mobilization of calcium & $4.0 \times 10^{-7}$ & 19 \\
\hline Adhesion of cells & $7.8 \times 10^{-7}$ & 29 \\
\hline Cell death & $1.1 \times 10^{-6}$ & 66 \\
\hline Tumorigenesis & $1.2 \times 10^{-6}$ & 72 \\
\hline Binding of cells & $2.9 \times 10^{-6}$ & 21 \\
\hline Viral elimination & $4.3 \times 10^{-6}$ & 3 \\
\hline Cancer & $4.9 \times 10^{-6}$ & 64 \\
\hline Inflammatory disorder & $5.3 \times 10^{-6}$ & 44 \\
\hline Cell growth & $5.6 \times 10^{-6}$ & 50 \\
\hline Cardiovascular disorder of lung & $1.4 \times 10^{-4}$ & 3 \\
\hline Perturbation of mitochondria & $2.7 \times 10^{-4}$ & 2 \\
\hline Inflammatory response & $3.4 \times 10^{-4}$ & 18 \\
\hline Asthma & $2.5 \times 10^{-3}$ & 9 \\
\hline Free radical scavenging & $3.1 \times 10^{-3}$ & 8 \\
\hline
\end{tabular}

In addition, in the cell proliferation category there were 27 smoking-correlated genes related to lymphocyte proliferation, all but three of which were negatively correlated $\left(\mathrm{p}=5.2 \times 10^{-7}\right)$. Twenty-one inflammatory response associated genes were significantly correlated with smoking $\left(\mathrm{p}=3.4 \times 10^{-4}\right)$, including fourteen that were negatively correlated $(A D A, A D R B 2, C 3, C D 38, C H S T 2$, FASLG, GLNY, GZMA, IL18RAP, KLRG1, PIK3CG, PTGDR, PTPN6, SPON2 and TNFRSF14) and seven that were positively correlated with smoking (CLEC5A,IGHE, PLA2G7, RNASE2, S100A8, S100A12 and SERPINF1).

There was evidence of overrepresentation of cancer related functional assignments, including 72 smokingcorrelated genes that were associated with tumorigenesis $\left(\mathrm{p}=1.2 \times 10^{-6}\right), 23$ of which were positively correlated with smoking and 49 negatively correlated genes. There were also 64 genes associated with cancer $\left(\mathrm{p}=4.9 \times 10^{-6}\right)$, 21 positively correlated with smoking $(A C O X 2, A Q P 3$, CANX, CLEC10A, CYP1B1, EBI2, EPB41L3, GPR177, IGL@, IL13RA1, LDHA, MGST1, MMP25, MTHFD2, NRG1, PAICS, S100A8, SERPINF1, TFDP1, UGCG and $V C A N)$ and 43 negatively correlated genes (ABCB1, $A D A, \quad A D R B 2, \quad A K R 1 C 3, \quad A R I H 2, \quad A X I N 1, \quad C 3$, CACNA2D2, CD247, CDKN1C, CST7, CTSC, CTSL1,
EBF4, FASLG, FCGR3A, GZMA, HMOX1, IL2RB, KLRK1, MT2A, NCAM1, ND3, NEURL, PALLD, PGLYRP2, PIK3CG, PODN, PPP2R2B, PRF1, PRSS23, PTGDS, PTPN6, RASSF1, RHOC, RRAS, SLC1A7, SSBP3, TGFBR3,TRA@,TRG@,TTC38 and UBE2C).

In relation to respiratory-relevant pathologies, there were three genes associated with lung related cardiovascular disorder that were all negatively correlated with smoking (FASLG, HMOX1, PRF1; $\mathrm{p}=1.4 \times 10^{-4}$ ) and nine genes involved in asthma $\left(\mathrm{p}=5.9 \times 10^{-4}\right)$ including six negatively correlated with smoking (ADRB2, CX3CR1, GZMB, HMOX1, PTGDR and TNFRSF8) and three positively correlated (IGHE, NRG1 and PLA2G7).

There was also some over-representation of free-radical related functional assignments, including two genes involved in mitochondrial perturbation (GZMB and PRF1; $\left.\mathrm{p}=2.7 \times 10^{-4}\right)$ and eight genes associated with free radical scavenging $\left(\mathrm{p}=3.1 \times 10^{-3}\right)$; seven negatively correlated (FASLG, GZMA, GZMB, HMOX1, PIK3CG, PRF1, $R R A S)$ and one (IGHE) positively correlated with cigarette smoking.

There were five significant canonical pathway categories of smoking correlated genes that also relate well to known smoking pathologies, shown in Table 2. The most 
significant of these canonical pathways assignments was for eleven genes involved in the natural killer cell signaling pathway, all of which were negatively correlated with smoking (CD247, CD300A, FCGR3A, FCGR3B, KLRB1, KLRD1, KLRK1, PIK3CG, PTPN6, RRAS and SH2D1B; $\mathrm{p}$ $=7.9 \times 10^{-7}$ ).

There were ten genes involved in the xenobiotic metabolism signaling pathway in our dataset $\left(\mathrm{p}=5.9 \times 10^{-3}\right)$, eight negatively correlated (ABCB1,CHST2, CHST12, HMOX1, PIK3CG, PPP2R2B, PPP2R5A and RRAS) and two that were positively correlated with smoking (CYP1B1 and MGST1). There were also four smokingcorrelated genes in the related metabolism of xenobiotics by cytochrome P450 canonical pathway $\left(\mathrm{p}=9.6 \times 10^{-3}\right)$, including three positively correlated (CSGALNACT1, $C Y P 1 B 1$ and MGST1) and one negatively correlated with smoking (AKR1C3). Cigarette smoke is a significant source of xenobiotics (chemicals foreign to the biological system) and these potentially damaging compounds are detoxified through such pathways.

\section{Network Analysis}

There were 243 smoking-correlated genes with known connectivity information from published literature stored in the Ingenuity Knowledge Base. We used this published interaction information to determine whether our smoking-correlated expression signatures are tightly connected at the molecular level. This analysis is therefore restricted to interactions derived from the published literature and does not identify novel interactions between these genes. Network analysis was used to determine whether our smoking-correlated expression signatures are tightly connected at the molecular level, based only on these known interactions, by generating graphical representations of the interactions between genes and/or gene products in our dataset. Network analysis was used to connect 49 of the smoking correlated genes into a single network of gene/gene product interaction (Figure 1). This network includes a clear sub-network of 28 genes known to be involved in immune and inflammatory response (outlined in orange). Given their relevance to cigarette smoke exposure, we included the external toxi- cants nicotine and reactive oxygen species in the network.

\section{Genetic and Environmental Correlations of Expression with Smoking Behavior}

Observed correlations between smoking behavior and a given gene's expression level may be due to the causal environmental effect of smoking on expression, the shared genetic determinants that jointly influence transcription level and the propensity to smoke, or a combination of these two influences. In order to assess the relative importance of environmental versus genetic sources of phenotypic covariation between expression levels and smoking status, we performed bivariate quantitative genetic analysis to decompose the observed phenotypic correlation of the 50 most significantly correlated transcripts. Because of our large pedigree-based study design, it is possible to directly estimate both the genetic and environmental correlations between expression levels and smoking status. Our results, documented in Additional File 2, clearly indicate that for all but one of the transcripts tested (98\%) we saw no evidence for genetic correlation between smoking behavior and expression levels as would be expected if the observed correlation was the result of a genetic predisposition to smoking behavior. Given the strength of the estimated environmental component of covariation between smoking behavior and expression levels, our observed correlations most likely reflect the causal influence of smoking on transcription levels, which suggests that smoking is acting as a direct environmental mediator of transcription.

\section{Discussion}

This study is the largest investigation of gene expression alterations in response to cigarette smoke exposure in human subjects in vivo to date. The results clearly reveal the broad influence of smoking, as an environmental influence, on the lymphocyte transcriptome. The results include a wide-ranging negative influence on the immune system, and strong involvement in a range of other relevant functional categories including cancer, cell death and xenobiotic metabolism. It is likely that this observed

Table 2: The most highly significant canonical pathway assignments for the set of smoking-correlated genes

\begin{tabular}{ll}
\hline Canonical pathway & P-value \\
\hline Natural killer cell signaling & $7.9 \times 10^{-7}$ \\
$\begin{array}{l}\text { CTLA4 Signaling in cytotoxic T } \\
\text { lymphocytes }\end{array}$ & $1.2 \times 10^{-4}$ \\
$\begin{array}{l}\text { SAPK/JNK signaling } \\
\text { Xenobiotic metabolism signaling } \\
\text { Metabolism of xenobiotics by cytochrome } \\
\text { P450 }\end{array}$ & $5.7 \times 10^{-3}$ \\
\hline
\end{tabular}




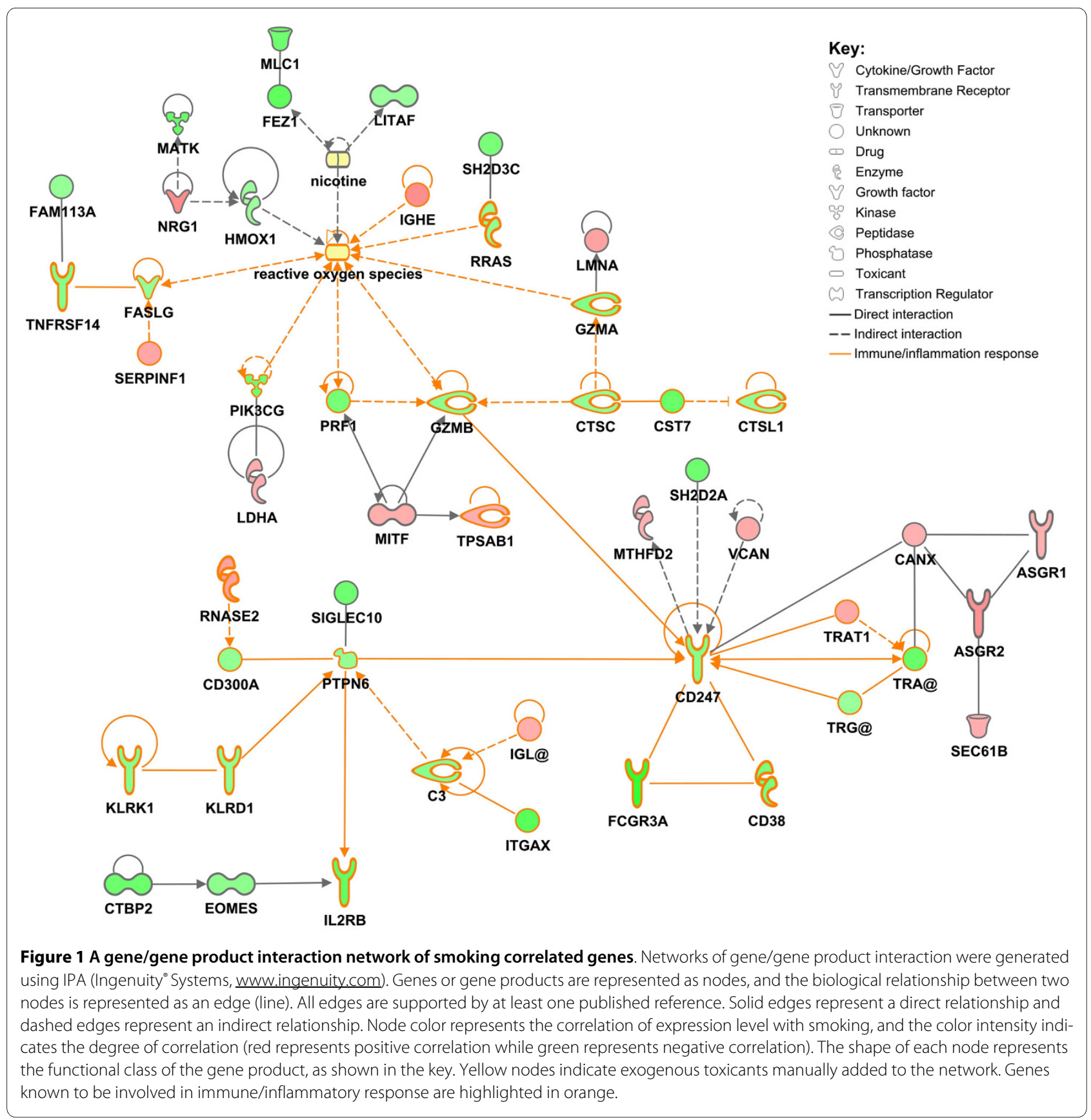

effect of smoking on transcription has larger implications for human disease risk, especially in relation to the increased risk of a wide variety of cancers throughout the body as a result of cigarette smoke exposure.

Peripheral lymphocytes appear to be an excellent surrogate tissue for investigating the effect of smoking on health by transcriptomic epidemiology. Not only are they one of the most readily and easily available tissues for gene expression analysis, they have also been shown to be a good surrogate for other tissue types in the case of environmental exposures, such as cigarette smoke, polyaro- matic hydrocarbons and radiation [30-32]. The biological value of the lymphocyte as a surrogate for another more directly involved tissue (e.g., lung tissue) does not require that similar expression patterns be expressed between lymphocytes and the more focal tissue. It merely requires that, for a given gene, there is a phenotypic correlation between expression levels in the two tissues, which is much less restrictive than the requirements of similar absolute patterns of expression. Absolute levels are immaterial to our central hypothesis. It is highly likely that the regulatory machinery across tissue types is 
altered by mechanisms of attenuation or amplification which would lead to dramatically different absolute levels but still generate correlations between tissue types. If the attenuation/amplification mechanism has a linear component, then correlations will obligately result. Therefore, any regulatory feature that is shared across tissues will generate a correlation between tissues. Thus, a gene may be very highly expressed in one tissue and lowly expressed in a different tissue and still exhibit correlation between tissues. Such correlations are beginning to be demonstrated across a number of tissues [26].

Lymphocytes may be directly relevant for assessing the damaging effects of cigarette smoke. Thousands of cigarette smoke constituents are rapidly absorbed into the bloodstream, through the pulmonary alveoli, where they rapidly achieve systemic distribution. Oxidative damage and polycyclic hydrocarbon adducts have been readily detected, not only the respiratory epithelium and other first sites of exposure in cigarette smokers, but also in the blood and peripheral lymphocytes [33,34]. In addition, of the 47,289 transcripts interrogated in our study, we were able to significantly detect 20,413 expressed transcripts in lymphocytes, including both known and predicted genes [24]. This is an impressive level of diversity for any tissue, and allows the flexibility to identify signatures of gene expression correlated with a range of traits. Finally, since smoking is a major risk factor for a wide variety of cancers and diseases in a range of tissue types, it is important to understand its influence at the gene level outside of a selected cancer model.

All of the most significant functional groupings of smoking correlated genes identified in this study are directly relevant to well known smoking related disease processes. Cell death and proliferation, immune response, cancer, inflammatory disease and xenobiotic metabolism are all relevant groupings for smoking correlated genes, given known smoking related pathologies. However, the extent of these relevant groupings and the number of correlated genes whose expression is influenced by smoke exposure within each group is striking.

Various aspects of depressed immune function have been well documented in smokers [9,35-40]. We identified sets of smoking correlated genes corresponding to immune system components that fit with this profile of wide-spread immune alteration and suppression. For example, all 17 genes associated with cell cytotoxicity were negatively correlated with smoking; of the 29 genes involved in immune response (Table 1), the expression levels of 23 were negatively correlated with smoking; and of the sixteen genes involved in inflammatory response, twelve were negatively correlated with smoking. All eleven genes in the natural killer (NK) cell signaling pathway (Table 2), involved in cytotoxicity and cytokine secretion, were also negatively correlated with smoking.
This wide-ranging negative influence on the immune system is one of the clearest pictures to emerge from our transcriptional profiling of smoking and gene expression. This comprehensive influence on immune related gene expression may go a long way towards explaining the processes behind the depressed immune system related pathologies exhibited by smokers.

As mentioned above, the expression levels of eleven genes in the natural killer (NK) cell signaling pathway were negatively correlated with smoking (CD247, CD300A, FCGR3A, FCGR3B, KLRB1, KLRD1, KLRK1, PIK3CG, PTPN6, RRAS and SH2D1B). NK cells are lymphocytes of the innate immune system involved in early defense against foreign cells and stressed autologous cells, and their cytotoxic activity is known to be decreased in smokers [36]. In addition, NK cell tumor immune surveillance was recently shown to be decreased in response to smoke exposure in a murine lung metastatic tumor model [41]. Our findings corroborate this negative influence of cigarette smoke on NK cell activity, and reveal some of the gene level alterations that may influence NK cells in smokers.

Another striking finding of this study was the over-representation of functional groupings relevant to cancer and cancer relevant processes such as cell death, proliferation and signaling. Cigarette smoking is a recognized risk factor for a wide variety of cancers, not only at the sites of contact such as the lungs and esophagus, but also throughout the body such as pancreatic, kidney, colon and bladder cancer. Correlations between expression of genes in lymphocytes and cigarette smoking in this study provide insight into the cancer relevant biological processes occurring throughout the body in response to smoke exposure, and hopefully serve to highlight the complexity of these processes.

We constructed a complex network based on published interaction between genes and/or gene products for 49 of the transcripts that were significantly correlated with smoking (Figure 1) including a clear sub-network of 28 genes known to be involved in immune and inflammatory response (outlined in orange). A portion of this network is also clustered around the exogenous toxicants nicotine and reactive oxygen species, with most of these genes involved in xenobiotic metabolism and free radical scavenging (FASLG, GZMA, GZMB, HMOX1, IGHE, PIK3CG, PRF1 and RRAS). The network displayed in Figure 1 clearly reveals the massive scale of influence that cigarette smoke, as an environmental variable, exerts over the lymphocyte transcriptome; however we are unable to determine which genes are directly influenced by smoke exposure, given that alteration of the expression level of one gene can alter the transcription of other genes in these networks. These interactions are made even more complex by the diversity of cigarette smoke components, 
and the variability of this constitution, in that we cannot identify which component(s) of cigarette smoke are exerting the most influence over gene expression. In addition, the network analysis is dependent on published connectivity information and as such cannot fully reflect the complexity of the interactions or reveal any novel connectivity. The literature reported interaction directionality is also of limited use in interpreting the empirical data, since our results reflect the state of the whole living organism. The observed correlations may reflect a large number of mediating factors including complex feedback loops that could easily be missed using classical in vitro cell-based models of cause and effect. However, this network does show that the genes whose expression is altered in response to cigarette smoke are tightly connected at the molecular level, and gives some indication of the pathways through which cigarette smoke influences known smoking related pathologies such as inflammation.

Although smoking has long-term adverse effects, cessation has some immediate, as well as long-term benefits, which may be due to a reversal of these transcriptomic alterations. Many of the negative health effects have been shown to be reversed or at least improved soon after cessation $[4,41,42]$, for example elevation of NK activity (which is suppressed in smokers) is detectable within one month of smoking cessation [43]. This further supports the implication that at least part of the NK cell activity suppression is due to gene-level alterations in expression induced by smoke exposure, which may be reversed as the exposure is removed. However, a study comparing transcriptional profiles of 34 smokers, 23 non-smokers and 18 former smokers revealed that, while the majority of smoke exposure related gene expression alterations return to normal in former smokers, there were a set of transcripts that appeared to retain altered expression patterns two-years after smoking cessation [16]. Similarly, Beane et al. showed that $16 \%$ of the 175 differentially expressed genes identified in airway epithelial cells between smokers and non-smokers were irreversibly altered in former smokers [12]. Therefore, while it appears that the majority of gene expression alterations attributable to smoke exposure may be reversible, there may also be a subset of genes for which the expression changes are permanent or at least altered in the long term.

This study has some intrinsic limitations that should be noted. The study was conducted in a population of Mexican American individuals and it is difficult to determine what proportion of the expression changes would be replicated in other populations. While results obtained from within a subset of the population may not necessarily be relevant to all subsets of the population, in general it is highly likely that the majority of genes whose expression is altered in Mexican American individuals in response to smoke exposure are the same genes susceptible to alteration in other population groups. Transcriptional alterations are likely more robust to population differences than studies of individual genetic variants. However, a subset of the genes identified in this study may only be relevant to the Mexican American population. It is also important to note that, while not likely, it is possible that cigarette smoking is a surrogate for some other influence that is initiating the transcriptional alterations in this study.

\section{Conclusions}

The results of this investigation offer insights into cigarette-smoke related pathological processes by determining its influences at the gene expression level. Never before has such a clear link between smoking and transcriptomics been revealed, and the scale at which exposure to cigarette smoke appears to influence the expression levels of our genes is sobering.

\section{Additional material}

\section{Additional file 1 Full details of the smoking-correlated genes signifi- cant at an FDR of $\mathbf{0 . 0 5}$. Includes gene information, correlation of expres- sion with smoking and the allocation of each gene into the functional assignments and canonical pathways from Ingenuity Pathways Analysis described in Tables 1 and 2 . \\ Additional file $\mathbf{2}$ Bivariate analysis results for the fifty most significant smoking-correlated transcripts. *Indicates there were multiple signifi- cant transcripts for this gene.}

\section{Competing interests}

The authors declare that they have no competing interests.

\section{Authors' contributions}

MCM, LA, JWM, EKM and JB designed and instigated the study including the inception and maintenance of the San Antonio Family Heart Study, phenotype collection and expression profiling. JEC and MPJ conducted the laboratory analyses including the expression profiling. JCC, HHHG, TDD, VPD, JWK, MCM and JB cleaned and/or analyzed the family, phenotype and expression data. JCC conducted the bioinformatic analyses. JCC and JB wrote the manuscript. All authors read and approved the final manuscript.

\section{Acknowledgements}

We wish to acknowledge the Azar and Shepperd families and ChemGenex Pharmaceuticals for supporting the transcriptional profiling, sequencing, genotyping and statistical analysis. The supercomputing facilities used for this work at the AT\&T Genomics Computing Center were supported in part by a gift from the SBC Foundation

Data collection was supported by a grant from the US National Institute for Heart, Lungs and Blood (HL045222). The SOLAR statistical genetics computer package is supported by a grant from the US National Institute of Mental Health (MH059490). The laboratory work was carried out in facilities that were constructed with support from the US National Center for Research Resources (RR013556).

\section{Author Details}

'Department of Genetics, Southwest Foundation for Biomedical Research, P.O. Box 760549, San Antonio, TX, USA and 2Menzies Research Institute, Private Bag 23, Hobart, TAS, Australia 
Received: 15 January 2010 Accepted: 15 July 2010

Published: 15 July 2010

\section{References}

1. Mathers CD, Loncar D: Projections of global mortality and burden of disease from 2002 to 2030. PLoS Med 2006, 3(11):e442.

2. Winniford MD: Smoking and cardiovascular function. J Hypertens Supp/ 1990, 8(5):S17-23.

3. U.S. Department of Health and Human Services: The Health Consequences of Smoking: A report of the Surgeon General. Atlanta, GA.: U.S. Department of Health and Human Services, Centers for Disease Control and Prevention, National Center for Chronic Disease Prevention and Health Promotion, Office on Smoking and Health; 2004.

4. Fagerstrom $\mathrm{K}$ : The epidemiology of smoking: health consequences and benefits of cessation. Drugs 2002, 62(Suppl 2):1-9.

5. Sherman CB: The health consequences of cigarette smoking. Pulmonary diseases. Med Clin North Am 1992, 76(2):355-375.

6. Newcomb PA, Carbone PP: The health consequences of smoking. Cancer. Med Clin North Am 1992, 76(2):305-331.

7. McBride PE: The health consequences of smoking. Cardiovascular diseases. Med Clin North Am 1992, 76(2):333-353.

8. Shopland DR: Tobacco use and its contribution to early cancer mortality with a special emphasis on cigarette smoking. Environ Health Perspect 1995, 103(Suppl 8):131-142.

9. Sopori M: Effects of cigarette smoke on the immune system. Nat Rev Immunol 2002, 2(5):372-377.

10. Baum SL, Anderson IGM, Baker RR, Murphy DM, Rowlands CC: Electron spin resonance and spin trap investigation of free radicals in cigarette smoke: development of a quantification procedure. Anal Chim Acta 2003, 481(1):1-13

11. Lodovici M, Luceri C, De Filippo C, Romualdi C, Bambi F, Dolara P: Smokers and passive smokers gene expression profiles: correlation with the DNA oxidation damage. Free Radic Biol Med 2007, 43(3):415-422.

12. Beane J, Sebastiani P, Liu G, Brody JS, Lenburg ME, Spira A: Reversible and permanent effects of tobacco smoke exposure on airway epithelial gene expression. Genome Biol 2007, 8(9):R201.

13. Ryder Ml, Hyun W, Loomer P, Haqq C: Alteration of gene expression profiles of peripheral mononuclear blood cells by tobacco smoke: implications for periodontal diseases. Oral Microbiol Immunol 2004, 19(1):39-49

14. Heguy A, O'Connor TP, Luettich K, Worgall S, Cieciuch A, Harvey BG, Hackett NR, Crystal RG: Gene expression profiling of human alveolar macrophages of phenotypically normal smokers and nonsmokers reveals a previously unrecognized subset of genes modulated by cigarette smoking. J Mol Med 2006, 84(4):318-328

15. Lampe JW, Stepaniants SB, Mao M, Radich JP, Dai H, Linsley PS, Friend SH, Potter JD: Signatures of environmental exposures using peripheral leukocyte gene expression: tobacco smoke. Cancer Epidemiol Biomarkers Prev 2004, 13(3):445-453.

16. Spira A, Beane J, Shah V, Liu G, Schembri F, Yang X, Palma J, Brody JS Effects of cigarette smoke on the human airway epithelial cell transcriptome. Proc Natl Acad Sci USA 2004, 101(27):10143-10148.

17. van Leeuwen DM, van Agen E, Gottschalk RW, Vlietinck R, Gielen M, van Herwijnen MH, Maas LM, Kleinjans JC, van Delft JH: Cigarette smokeinduced differential gene expression in blood cells from monozygotic twin pairs. Carcinogenesis 2006

18. Buttner P, Mosig S, Funke H: Gene expression profiles of T lymphocytes are sensitive to the influence of heavy smoking: a pilot study. Immunogenetics 2007, 59(1):37-43

19. Chari R, Lonergan KM, Ng RT, Macaulay C, Lam WL, Lam S: Effect of active smoking on the human bronchial epithelium transcriptome. BMC Genomics 2007, 8(1):297.

20. Sridhar S, Schembri F, Zeskind J, Shah V, Gustafson AM, Steiling K, Liu G, Dumas YM, Zhang X, Brody JS, et al:: Smoking-induced gene expression changes in the bronchial airway are reflected in nasal and buccal epithelium. BMC Genomics 2008, 9:259.

21. Zhang X, Sebastiani P, Liu G, Schembri F, Dumas YM, Langer EM, Alekseyev Y, O'Connor GT, Brooks DR, Lenburg ME, et al: Similarities and differences between smoking-related gene expression in nasal and bronchial epithelium. Physiol Genomics 2009.
22. MacCluer JW, Stern MP, Almasy L, Atwood LA, Blangero J, Comuzzie AG, Dyke B, Haffner SM, Henkel RD, Hixson JE, et al: Genetics of atherosclerosis risk factors in Mexican Americans. Nutr Rev 1999, 57(5 Pt 2):S59-65.

23. McPeek MS, Sun L: Statistical tests for detection of misspecified relationships by use of genome-screen data. Am J Hum Genet 2000, 66(3):1076-1094.

24. Göring HH, Curran JE, Johnson MP, Dyer TD, Charlesworth J, Cole SA, Jowett JB, Abraham LJ, Rainwater DL, Comuzzie AG, et al:: Discovery of expression QTLs using large-scale transcriptional profiling in human lymphocytes. Nat Genet 2007

25. Almasy L, Blangero J: Multipoint quantitative-trait linkage analysis in general pedigrees. Am J Hum Genet 1998, 62(5):1198-1211.

26. Benjamini Y, Hochberg Y: Controlling the False Discovery Rate - a Practical and Powerful Approach to Multiple Testing. J Roy Stat Soc B Met 1995, 57(1):289-300

27. Williams JT, Van Eerdewegh P, Almasy L, Blangero J: Joint multipoint linkage analysis of multivariate qualitative and quantitative traits. I. Likelihood formulation and simulation results. Am J Hum Genet 1999, 65(4):1134-1147.

28. Perez-Stable EJ, Marin BV, Marin G, Brody DJ, Benowitz NL: Apparent underreporting of cigarette consumption among Mexican American smokers. Am J Public Health 1990, 80(9):1057-1061.

29. Swan GE, Lessov-Schlaggar CN, Bergen AW, He Y, Tyndale RF, Benowitz $\mathrm{NL}$ : Genetic and environmental influences on the ratio of 3 'hydroxycotinine to cotinine in plasma and urine. Pharmacogenet Genomics 2009, 19(5):388-398

30. Van Schooten FJ, Godschalk RW, Breedijk A, Maas LM, Kriek E, Sakai H, Wigbout G, Baas P, Van't Veer L, Van Zandwijk N: 32P-postlabelling of aromatic DNA adducts in white blood cells and alveolar macrophages of smokers: saturation at high exposures. Mutat Res 1997, 378(12):65-75.

31. Rockett JC, Burczynski ME, Fornace AJ, Herrmann PC, Krawetz SA, Dix DJ: Surrogate tissue analysis: monitoring toxicant exposure and health status of inaccessible tissues through the analysis of accessible tissues and cells. Toxicol Appl Pharmacol 2004, 194(2):189-199.

32. Wiencke JK, Kelsey KT, Varkonyi A, Semey K, Wain JC, Mark E, Christiani DC: Correlation of DNA adducts in blood mononuclear cells with tobacco carcinogen-induced damage in human lung. Cancer Res 1995 55(21):4910-4914.

33. Kiyosawa H, Suko M, Okudaira H, Murata K, Miyamoto T, Chung MH, Kasai $\mathrm{H}$, Nishimura S: Cigarette smoking induces formation of 8hydroxydeoxyguanosine, one of the oxidative DNA damages in human peripheral leukocytes. Free Radic Res Commun 1990, 11(1-3):23-27.

34. Tang D, Santella RM, Blackwood AM, Young TL, Mayer J, Jaretzki A, Grantham S, Tsai WY, Perera FP: A molecular epidemiological casecontrol study of lung cancer. Cancer Epidemiol Biomarkers Prev 1995, 4(4):341-346.

35. Phillips B, Marshall ME, Brown S, Thompson JS: Effect of smoking on human natural killer cell activity. Cancer 1985, 56(12):2789-2792.

36. Zeidel A, Beilin B, Yardeni I, Mayburd E, Smirnov G, Bessler H: Immune response in asymptomatic smokers. Acta Anaesthesiol Scand 2002, 46(8):959-964.

37. Moszczynski P, Zabinski Z, Moszczynski P Jr, Rutowski J, Slowinski S, Tabarowski Z: Immunological findings in cigarette smokers. Toxicol Lett 2001, 118(3):121-127.

38. Geng Y, Savage SM, Razani-Boroujerdi S, Sopori ML: Effects of nicotine on the immune response. II. Chronic nicotine treatment induces T cell anergy. J Immunol 1996, 156(7):2384-2390.

39. McCue JM, Link KL, Eaton SS, Freed BM: Exposure to cigarette tar inhibits ribonucleotide reductase and blocks lymphocyte proliferation. J Immunol 2000, 165(12):6771-6775.

40. Sopori ML, Kozak W: Immunomodulatory effects of cigarette smoke. J Neuroimmunol 1998, 83(1-2):148-156.

41. Lu LM, Zavitz CC, Chen B, Kianpour S, Wan Y, Stampfli MR: Cigarette smoke impairs NK cell-dependent tumor immune surveillance. Immunol 2007, 178(2):936-943.

42. Willemse BW, Postma DS, Timens W, ten Hacken NH: The impact of smoking cessation on respiratory symptoms, lung function, airway hyperresponsiveness and inflammation. Eur Respir J 2004 23(3):464-476 
43. Meliska CJ, Stunkard ME, Gilbert DG, Jensen RA, Martinko JM: Immune function in cigarette smokers who quit smoking for 31 days. J Allergy Clin Immunol 1995, 95(4):901-910.

\section{Pre-publication history}

The pre-publication history for this paper can be accessed here: http://www.biomedcentral.com/1755-8794/3/29/prepub

doi: 10.1186/1755-8794-3-29

Cite this article as: Charlesworth et al., Transcriptomic epidemiology of smoking: the effect of smoking on gene expression in lymphocytes BMC Medical Genomics 2010, 3:29

Submit your next manuscript to BioMed Central and take full advantage of:

- Convenient online submission

- Thorough peer review

- No space constraints or color figure charges

- Immediate publication on acceptance

- Inclusion in PubMed, CAS, Scopus and Google Scholar

- Research which is freely available for redistribution

Submit your manuscript at www.biomedcentral.com/submit 